\title{
Malignant cardiac metastasis from breast cancer: Imaging contribution to surgical attitude
}

\author{
Victor J. Ovejero-Gomez ${ }^{1^{*}}$, L. Martin-Cuesta ${ }^{2}$, V. Alija $^{3}$, J. Villalba $^{4}$, J. Rodríguez-Cabello $^{2}$, \\ J. Perez ${ }^{5}$, J. M. Bajo-Arenas ${ }^{4}$ \\ ${ }^{1}$ Department of Surgery, Hospital Sierrallana de Torrelavega, Torrelavega, Spain; ${ }^{*}$ Corresponding Author: vovejerohcas@msn.com \\ ${ }^{2}$ Department of Radiology, Hospital Sierrallana de Torrelavega, Torrelavega, Spain \\ ${ }^{3}$ Department of Medical Oncology, Hospital Sierrallana de Torrelavega, Torrelavega, Spain \\ ${ }^{4}$ Department of Gynaecology, Hospital Sierrallana de Torrelavega, Torrelavega, Spain \\ ${ }^{5}$ Department of Cardiology, Hospital Sierrallana de Torrelavega, Torrelavega, Spain
}

Received 30 July 2013; revised 28 August 2013; accepted 30 September 2013

Copyright (c) 2013 Victor Ovejero et al. This is an open access article distributed under the Creative Commons Attribution License, which permits unrestricted use, distribution, and reproduction in any medium, provided the original work is properly cited.

\begin{abstract}
Metastasic cardiac disease from the breast is rarely diagnosed in the lifetime. It has a poor prognosis and limited management. Both echocardiography and computerized tomography (CT) should be the first imaging studies in suspicion of this entity. Other diagnostic methods should be based on the possibilities of treatment although a histopathological analysis of the metastasic mass is needed to confirm the diagnosis. Magnetic resonance imaging (MRI) could be useful to complete a morphological and functional evaluation in case of surgical removal.
\end{abstract}

Keywords: Cancer; Breast; Metastasis; Cardiac; Imaging

\section{INTRODUCTION}

The most common malignant neoplasms that metastasize to the heart are lung, breast and esophageal cancers, lymphoma, leukaemia and melanoma [1], which have the highest prevalence of cardiac metastasis per 100 cases of any neoplasm.

Malignant cardiac metastasis from breast cancer usually appears late in the context of a wider involvement and only $10 \%$ of them have symptoms. They frequently contribute to the mechanism of death.

Advances in imaging techniques have improved clinical diagnosis despite its poor prognosis. The treatment includes a combination of surgery, radiotherapy and chemotherapy.

The value of radiological findings could be helpful to guide a therapeutic approach.

We present a female patient diagnosed with breast carcinoma, who suffered from asymptomatic regional recurrence and metastasic involvement of the right side of the heart. Our aim is to analyze the current imaging methods in cardiac metastasic neoplasms according to both their cost-effective and therapeutic profitability.

\section{CASE REPORT}

A 54-year-old woman with previous medical history of hypertension and hypercholesterolemia underwent tumorectomy with negative margins and negative sentinel lymph node for a left-breast ductal carcinoma with hormone receptor-positive. Six months later, she presented palpable axillary nodes on physical examination. A left-axillary lymphadenectomy was carried out and the histologic specimen demonstrated two positive lymph nodes over the fifteen removed. Postoperative, the patient was staged as $\mathrm{pT}_{2} \mathrm{pN}_{1} \mathrm{M}_{0}$.

It was indicated both adjuvant chemotherapy and radiotherapy. In the clinical follow-up, the patient touched a node on her surgical scar and a new biopsy indicated malignancy. An analysis of biomarkers showed a high value of Ca 15.3 (181.6 U/L, normal rate < 25) and CEA $(29.2 \mu \mathrm{g} / \mathrm{L}$, normal rate $<3.5)$.

A simple mastectomy was performed and she was treated with hormone agents.

She was in complete remission for a short-term due to another asymptomatic local soft-tissue recurrence and proposed for surgical approach.

A CT-scan showed a local malignant progression onto thoracic wall and a myocardial mass (Figures 1 and 2) with repolarization changes in EKG, which were not evident on radiological and clinical studies before. 


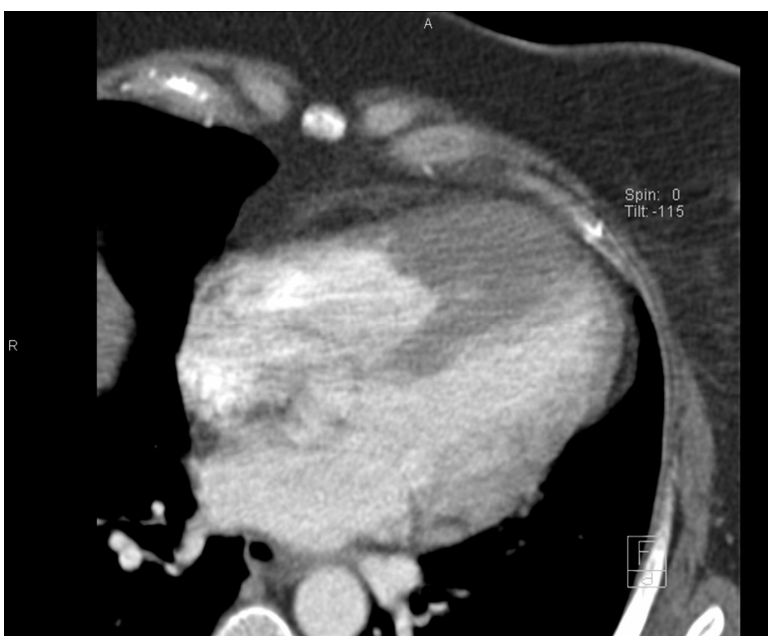

Figure 1. Axial contrast material-enhanced CT scan shows myocardial mass with endocardial extension in the anterior wall and septum of the right ventricle.

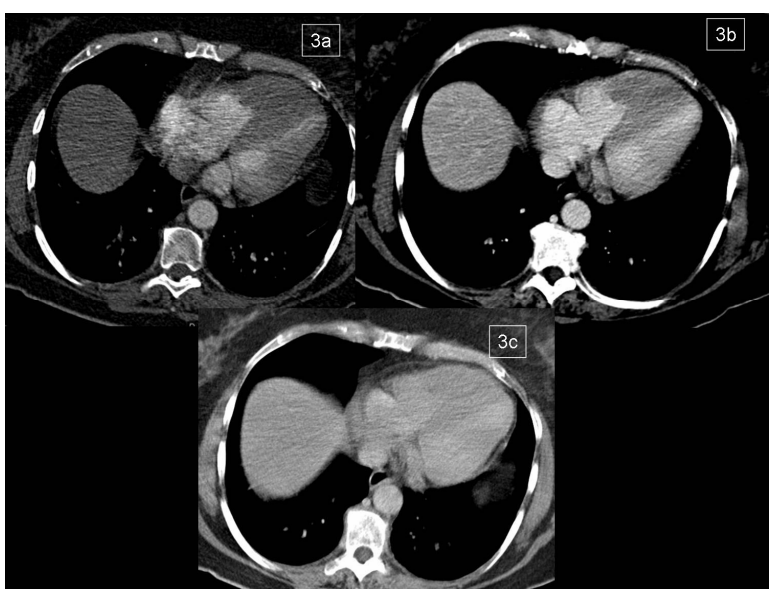

Figure 2. Triphasic helical CT. Axial image. Arterial (3a), venous (3b) and delayed (3c) phases show a low attenuation mass in the right ventricle myocardium with homogeneous subtle enhancement and delayed wash-out.

An echocardiogram (Figure 3) showed a partial myocardial wall achinesia in right ventricle and one endoluminal lesion with a broad myocardial infiltration as it could be demonstrated by a further magnetic resonance imaging (Figure 4). A biopsy was taken but inflammation could only be evidenced on insufficient sample.

She underwent palliative chemotherapy and surgical approach was ruled out.

On the last follow-up two months later, there was a progression of the disease. Shortly afterwards, our patient died from cardiogenic shock due to massive involvement of the heart.

\section{DISCUSSION}

Breast cancer is the second most common cause of

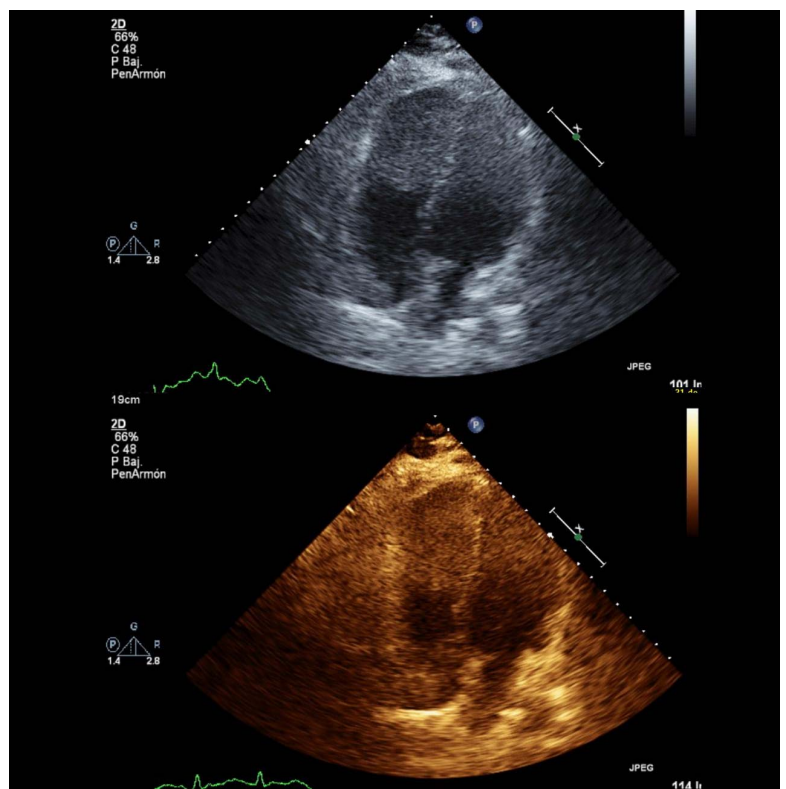

Figure 3. Echocardiography in this patient shows akinetic right ventricle with a space-occupying lesion suggestive of infiltration of medioapical segments.

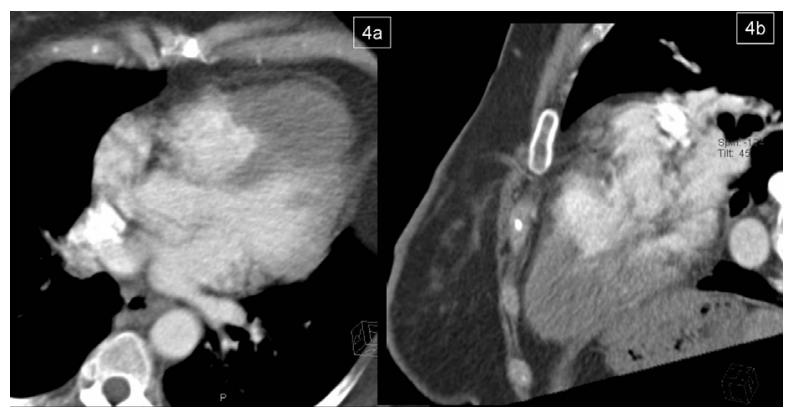

Figure 4. Axial (4a) and sagital (4b) MPR images display the mass affecting the right ventricle myocardium with endocardial growth and protrusion along the cardiac border.

cancer-related death in women, behind lung carcinoma. The thorax is a common site of mammary metastasis as both soft-tissue recurrence and lung metastasis. Nonetheless, the heart is often involved in patient lifetime, although they are usually asymptomatic and found late, even at autopsy [2,3]. This feature is common to other metastasic tumours to the heart [1].

Most of breast carcinoma metastasizes to the right side of the heart by direct extention due to its close proximity and the first metastasized layers are usually pericardium and epicardium. Hematogenous and lymphatic pathways are uncommon due to the strong and metabolic peculiarities of myocardial striated muscle, and the fast blood flow through the heart and lymph flow moving away from the heart [4].

Some reported cases, similar to ours, were diagnosed as a late cardiac disease on the left ventricle after a mastectomy and pulmectomy which could support this 
sort of dissemination [5]. On the contrary, an early soft-tissue recurrence on a rib cage and a right ventricle mass seemed to suggest that this cancer was spread by a direct pathway.

In patients with clinical suspicious of cardiac metastases from breast, a first imaging study should be performed to evaluate the chest wall, lung, pleura and mediastinum to rule out other spreads which could modify the therapeutic strategy.

A focused imaging study on cardiac metastasis should be considered cost-effectiveness of the radiologic method due to the poor prognosis of the disease, even though the surgical removal is indicated.

Echocardiography is a noninvasive imaging study and the first diagnostic choice which could provide information about the size and location of the metastasic mass [6]. The cardiac CT scan and MRI could be useful to evaluate the local recurrence and cardiothoracic metastasis with regard to anatomical relations when a surgical removal has been planned.

In fact, some of these patients present their first recurrence either on the chest wall or lymph nodes. Pulmonary and cardiac metastasis will appear soon afterwards [7].

Nonetheless, CMR offers a better contrast resolution to distinguish among the mass, thrombus and blood flow artifact with typically low signal intensity on T1weighted images and higher signal intensity on T2weighted images unlike other cardiac metastasis as melanoma [8].

Nowadays, ultrasonography enables a better performance from a practical point of view: A histological sample can be obtained by transesophageal echo-guided biopsy instead of cardiac catheterism, and a 3-dimensional transthoracic echocardiography (3D-TTE) is able to show cardiac functional volumes and 3D images about tumoral mass' surface, type, location and anatomic relations with greater accuracy than 2D-TTE which underestimates the cardiac function and volume mass [9].

Positron emission tomography and other techniques of scintigraphy are imaging methods to confirm the malignancy and cardiac function although its contribution is limited [10].

In the postoperative follow-up for breast cancer, local recurrence should be evaluated primarily by physical examination and mamography, with a sensitivity of 79 and $45 \%$ respectively, although this imaging technique could be compromised by the presence of postoperative distorsion and the increased density of an irradiated breast. Ultrasonography could be more helpful thanks to a higher sensitivity, superior to $90 \%$.

However, computed tomography is the most sensitive examination to detect thoracic metastasis and regional recurrence. It should be the first choice in imaging methods because it allows a more accurate determination of recurrence and is able to demonstrate clinically unsuspected disease in $49 \%$ of patients.

The use of other imaging studies depends on the planned therapeutic management since its diagnosis is not a surgical contraindication by itself if this option is considered.

Most cardiac metastases from breast carcinoma are incurable and a palliative therapy based on a systemic chemotherapy only should be offered because radiotherapy is rarely helpful and indicated to relieve symptoms [11]. An echocardiography, CT or MRI and echoguided biopsy could be enough to make a decision in these cases.

Nonetheless, some patients who suffer from tamponade or obstruction of blood flow could undergo palliative emergency surgery although a lot of these cases have been reported with a high postoperative mortality due to cardiological complications [12].

This unforeseeable development of the natural history of the disease should not justify a previous detailed imaging study because the most common cause of death is used to being a cardiorespiratory arrest without any surgical chance.

A complete imaging study only should be indicated when a surgical removal is planned.

Radiological contribution should focus on the diagnosis and functional or morphological characterization of the heart by MRI and echocardiogram to support the therapeutic management decision.

\section{REFERENCES}

[1] Tesolin, M., Lapierre, C., Oligny, L., Bigras, J.L. and Champagne, M. (2005) Cardiac metastases from melanoma. Radiographics, 25, 249-253. http://dx.doi.org/10.1148/rg.251045059

[2] Jung, J.I., Kim, H.H., Park, S.H., Song, S.W., Chung, M.H., Kim, H.S., et al. (2004) Thoracic manifestations of breast cancer and its therapy. Radiographics, 24, 12691285. http://dx.doi.org/10.1148/rg.245035062

[3] Butany, J., Leong, S.W., Carmichael, K. and Komeda, M. (2005) A 30-year analysis of cardiac neoplasms at autopsy. Canadian Journal of Cardiology, 21, 675-680.

[4] Choi, P.W., Chul, N.K., Chang, S.H., Chang, W.I., Chang, Y.Y. and Hyun, M.C. (2009) Cardiac metastasis from colorectal cancer: A case report. World Journal of Gastroenterology, 15, 2675-2678. http://dx.doi.org/10.3748/wjg.15.2675

[5] Katalinic, D., Stern-Padovan, R., Ivanac, I., Aleric, I., Tentor, D., Nikolac, N., et al. (2013) Symptomatic cardiac metastases of breast cancer 27 years after mastectomy: A case report with literature review-Pathophysiology of molecular mechanisms and metastasic pathways, clinical aspects, diagnostic procedures and treatment modalities. World Journal of Surgical Oncology, 11, 14. 
http://dx.doi.org/10.1186/1477-7819-11-14

[6] Butany, J., Nair, V., Naseemuddin, A., Nair, G.M., Catton, C. and Yau, T. (2005) Cardiac tumours: Diagnosis and management. The Lancet Oncology, 6, 219-228. http://dx.doi.org/10.1016/S1470-2045(05)70093-0

[7] Pavithran, K., Doval, D.C, Ravi, S., Mittal, R. and Bapsy, P.P. (1997) Cardiac metastasis from carcinoma breast-A case report. Indian Journal of Medical Research, 51, 1517.

[8] Chiles, C., Woodard, P.K., Gutierrez, F.R. and Link, K.M. (2001) Metastasic involvement of the heart and pericardium: CT and MR imaging. Radiographics, 21, 439-449.

[9] Leja, M.J., Shah, D.J. and Reardon, M.J. (2011) Primary cardiac tumors. Texas Heart Institute Journal, 38, 261-
262.

[10] Kawase, T., Fujii, H., Nakahara, T., Shigematsu, N., Kubo, A. and Kosuda, S. (2009) Intense accumulation of Tc- 99 $\mathrm{m}$ MDP in pericardial metastasis from breast cancer. Clinical Nuclear Medicine, 34, 173-174. http://dx.doi.org/10.1097/RLU.0b013e31819672b9

[11] Reynen, K., Köckeritz, U. and Strasser, R.H. (2004) Metastases to the heart. Annals of Oncology, 15, 375-381. http://dx.doi.org/10.1093/annonc/mdh086

[12] Garg, N., Moorthy, N., Agrawal, S.K., Pandey, S. and Kumari, N. (2011) Delayed cardiac metastasis from phyllodes breast tumor presenting as cardiogenic shock. Texas Heart Institute Journal, 38, 441-444.

\section{ABBREVIATIONS}

CT: Computed tomography

MRI: Magnetic resonance imaging

Ca 15.3: Carbohydrate antigen 15.3

CEA: Carcinoembryonic antigen

EKG: Electrocardiogram

CMR: Cardiovascular magnetic resonance

3D-TTE: Three-dimensional transthoracic echocardiography 3D images: Tridimensional images

2D-TTE: Two-dimensional transthoracic echocardiography MPR: Multiplanar reconstruction. 